\title{
MULTI-OBJECTIVE MODEL CHECKING OF MARKOV DECISION PROCESSES *
}

\author{
KOUSHA ETESSAMI ${ }^{a}$, MARTA KWIATKOWSKA $^{b}$, MOSHE Y. VARDI $^{c}$, \\ AND MIHALIS YANNAKAKIS ${ }^{d}$
}

${ }^{a}$ LFCS, School of Informatics, University of Edinburgh, UK

e-mail address: kousha@inf.ed.ac.uk

${ }^{b}$ Computing Laboratory, Oxford University, UK

e-mail address: Marta.Kwiatkowska@comlab.ox.ac.uk

${ }^{c}$ Department of Computer Science, Rice University, USA

e-mail address: vardi@cs.rice.edu

${ }^{d}$ Department of Computer Science, Columbia University, USA

e-mail address: mihalis@cs.columbia.edu

ABSTRACT. We study and provide efficient algorithms for multi-objective model checking problems for Markov Decision Processes (MDPs). Given an MDP, $M$, and given multiple linear-time ( $\omega$-regular or LTL) properties $\varphi_{i}$, and probabilities $r_{i} \in[0,1], i=1, \ldots, k$, we ask whether there exists a strategy $\sigma$ for the controller such that, for all $i$, the probability that a trajectory of $M$ controlled by $\sigma$ satisfies $\varphi_{i}$ is at least $r_{i}$. We provide an algorithm that decides whether there exists such a strategy and if so produces it, and which runs in time polynomial in the size of the MDP. Such a strategy may require the use of both randomization and memory. We also consider more general multi-objective $\omega$-regular queries, which we motivate with an application to assume-guarantee compositional reasoning for probabilistic systems.

Note that there can be trade-offs between different properties: satisfying property $\varphi_{1}$ with high probability may necessitate satisfying $\varphi_{2}$ with low probability. Viewing this as a multi-objective optimization problem, we want information about the "trade-off curve" or Pareto curve for maximizing the probabilities of different properties. We show that one can compute an approximate Pareto curve with respect to a set of $\omega$-regular properties in time polynomial in the size of the MDP.

Our quantitative upper bounds use LP methods. We also study qualitative multiobjective model checking problems, and we show that these can be analysed by purely graph-theoretic methods, even though the strategies may still require both randomization and memory.

1998 ACM Subject Classification: G.3, F.2, F.3.1, F.4.1.

Key words and phrases: Markov Decision Processes, Model Checking, Multi-Objective Optimization.

* A preliminary version of this paper appeared in the Proceedings of the 13th International Conference on Tools and Algorithms for the Construction and Analysis of Systems (TACAS'07).

\section{님}

DOI:10.2168/LMCS-4 (4:8) 2008

(C) K. Etessami, M. Kwiatkowska, M. Y. Vardi, and M. Yannakakis (c) Creative Commons 

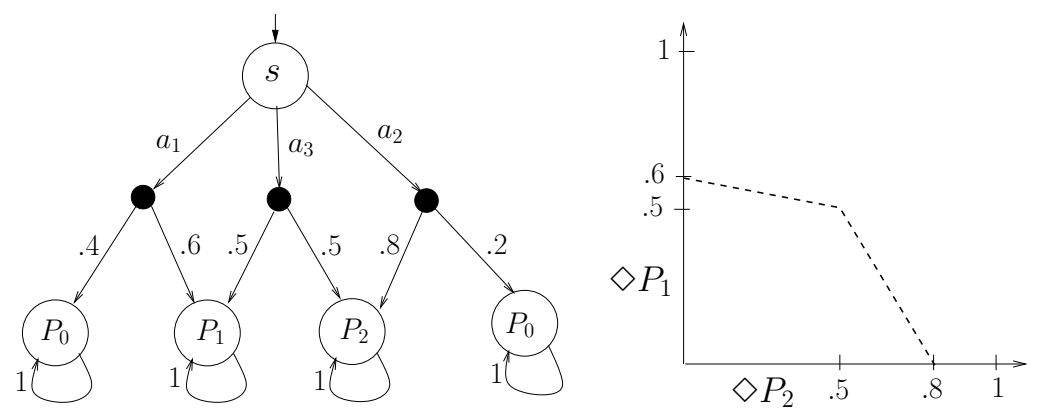

Figure 1: An MDP with two objectives, $\diamond P_{1}$ and $\diamond P_{2}$, and the associated Pareto curve.

\section{INTRODUCTION}

Markov Decision Processes (MDPs) are standard models for stochastic optimization and for modelling systems with probabilistic and nondeterministic or controlled behavior (see [Put94, Var85, CY95, CY98]). In an MDP, at each state, the controller can choose from among a number of actions, or choose a probability distribution over actions. Each action at a state determines a probability distribution on the next state. Fixing an initial state and fixing the controller's strategy determines a probability space of infinite runs (trajectories) of the MDP. For MDPs with a single objective, the controller's goal is to optimize the value of an objective function, or payoff, which is a function of the entire trajectory. Many different objectives have been studied for MDPs, with a wide variety of applications. In particular, in verification research linear-time model checking of MDPs has been studied, where the objective is to maximize the probability that the trajectory satisfies a given $\omega$-regular or LTL property (CY98, CY95, Var85]).

In many settings we may not just care about a single property. Rather, we may have a number of different properties and we may want to know whether we can simultaneously satisfy all of them with given probabilities. For example, in a system with a server and two clients, we may want to maximize the probability for both clients 1 and 2 of the temporal property: "every request issued by client $i$ eventually receives a response from the server", $i=1,2$. Clearly, there may be a trade-off. To increase this probability for client 1 we may have to decrease it for client 2 , and vice versa. We thus want to know what are the simultaneously achievable pairs $\left(p_{1}, p_{2}\right)$ of probabilities for the two properties. More specifically, we will be interested in the "trade-off curve" or Pareto curve. The Pareto curve is the set of all achievable vectors $p=\left(p_{1}, p_{2}\right) \in[0,1]^{2}$ such that there does not exist another achievable vector $p^{\prime}$ that dominates $p$, meaning that $p \leq p^{\prime}$ (coordinate-wise inequality) and $p \neq p^{\prime}$.

Concretely, consider the very simple MDP depicted in Figure 1. Starting at state $s$, we can take one of three possible actions $\left\{a_{1}, a_{2}, a_{3}\right\}$. Suppose we are interested in LTL properties $\diamond P_{1}$ and $\diamond P_{2}$. Thus we want to maximize the probability of reaching the two distinct vertices labeled by $P_{1}$ and $P_{2}$, respectively. To maximize the probability of $\diamond P_{1}$ we should take action $a_{1}$, thus reaching $P_{1}$ with probability 0.6 and $P_{2}$ with probability 0 . To maximize the probability of $\diamond P_{2}$ we should take $a_{2}$, reaching $P_{2}$ with probability 0.8 and $P_{1}$ with probability 0 . To maximize the sum total probability of reaching $P_{1}$ or $P_{2}$, we should take $a_{3}$, reaching both with probability 0.5. Now observe that we can also "mix" these pure strategies using randomization to obtain any convex combination of these three 
value vectors. In the graph on the right in Figure 1, the dotted line plots the Pareto curve for these two properties.

The Pareto curve $\mathcal{P}$ in general contains infinitely many points, and it can be too costly to compute an exact representation for it (see Section 21). Instead of computing it outright we can try to approximate it ([PY00]). An $\epsilon$-approximate Pareto curve is a set of achievable vectors $\mathcal{P}(\epsilon)$ such that for every achievable vector $r$ there is some vector $t \in \mathcal{P}(\epsilon)$ which "almost" dominates it, meaning $r \leq(1+\epsilon) t$.

In general, given a labeled $\operatorname{MDP} M, k$ distinct $\omega$-regular properties, $\Phi=\left\langle\varphi_{i}\right| i=$ $1, \ldots, k\rangle$, a start state $u$, and a strategy $\sigma$, let $\operatorname{Pr}_{u}^{\sigma}\left(\varphi_{i}\right)$ denote the probability that starting at $u$, using strategy $\sigma$, the trajectory satisfies $\varphi_{i}$. For a strategy $\sigma$, define the vector $t^{\sigma}=\left(t_{1}^{\sigma}, \ldots, t_{k}^{\sigma}\right)$, where $t_{i}^{\sigma}=\operatorname{Pr}_{u}^{\sigma}\left(\varphi_{i}\right)$, for $i=1, \ldots, k$. We say a value vector $r \in[0,1]^{k}$ is achievable for $\Phi$, if there exists a strategy $\sigma$ such that $t^{\sigma} \geq r$.

We provide an algorithm that given $\operatorname{MDP} M$, start state $u$, properties $\Phi$, and rational value vector $r \in[0,1]^{k}$, decides whether $r$ is achievable, and if so produces a strategy $\sigma$ such that $t^{\sigma} \geq r$. The algorithm runs in time polynomial in the size of the MDP. The strategies may require both randomization and memory. Our algorithm works by first reducing the achievability problem for multiple $\omega$-regular properties to one with multiple reachability objectives, and then reducing the multi-objective reachability problem to a multi-objective linear programming problem. We also show that one can compute an $\epsilon$-approximate Pareto curve for $\Phi$ in time polynomial in the size of the MDP and in $1 / \epsilon$. To do this, we use our linear programming characterization for achievability, and use results from [PY00] on approximating the Pareto curve for multi-objective linear programming problems.

We also consider more general multi-objective queries. Given a boolean combination $B$ of quantitative predicates of the form $\operatorname{Pr}_{u}^{\sigma}\left(\varphi_{i}\right) \Delta p$, where $\Delta \in\{\leq, \geq,<,>,=, \neq\}$, and $p \in[0,1]$, a multi-objective query asks whether there exists a strategy $\sigma$ satisfying $B$ (or whether all strategies $\sigma$ satisfy $B$ ). It turns out that such queries are not really much more expressive than checking achievability. Namely, checking a fixed query $B$ can be reduced to checking a fixed number of extended achievability queries, where for some of the coordinates $t_{i}^{\sigma}$ we can ask for a strict inequality, i.e., that $t_{i}^{\sigma}>r_{i}$. (In general, however, the number and size of the extended achievability queries needed may be exponential in the size of $B$.) A motivation for allowing general multi-objective queries is to enable assume-guarantee compositional reasoning for probabilistic systems, as explained in Section 2.

Whereas our algorithms for quantitative problems use LP methods, we also consider qualitative multi-objective queries. These are queries given by boolean combinations of predicates of the form $\operatorname{Pr}_{u}^{\sigma}\left(\varphi_{i}\right) \Delta b$, where $b \in\{0,1\}$. We give an algorithm using purely graph-theoretic techniques that decides whether there is a strategy that satisfies a qualitative multi-objective query, and if so produces such a strategy. The algorithm runs in time polynomial in the size of the MDP. Even for satisfying qualitative queries the strategy may need to use both randomization and memory.

In typical applications, the MDP is far larger than the size of the query. Also, $\omega-$ regular properties can be presented in many ways, and it was already shown in CY95] that the query complexity of model checking MDPs against even a single LTL property is 2EXPTIME-complete. We remark here that, if properties are expressed via LTL formulas, then our algorithms run in polynomial time in the size of the MDP and in 2EXPTIME in the size of the query, for deciding arbitrary multi-objective queries, where both the MDP and the query are part of the input. So, the worst-case upper bound is the same as with a single LTL objective. However, to keep our complexity analysis simple, we focus in this 
paper on the model complexity of our algorithms, rather than their query complexity or combined complexity.

Related work. Model checking of MDPs with a single $\omega$-regular objective has been studied in detail (see CY98, CY95, Var85]). In [CY98, Courcoubetis and Yannakakis also considered MDPs with a single objective given by a positive weighted sum of the probabilities of multiple $\omega$-regular properties, and they showed how to efficiently optimize such objectives for MDPs. They did not consider tradeoffs between multiple $\omega$-regular objectives. We employ and build on techniques developed in CY98.

Multi-objective optimization is a subject of intensive study in Operations Research and related fields (see, e.g., [Ehr05, Clí97]). Approximating the Pareto curve for general multi-objective optimization problems was considered by Papadimitriou and Yannakakis in [PY00]. Among other results, PY00] showed that for multi-objective linear programming (i.e., linear constraints and multiple linear objectives), one can compute a (polynomial sized) $\epsilon$-approximate Pareto curve in time polynomial in the size of the LP and in $1 / \epsilon$.

Our work is related to recent work by Chatterjee, Majumdar, and Henzinger ([CMH06]), who considered MDPs with multiple discounted reward objectives. They showed that randomized but memoryless strategies suffice for obtaining any achievable value vector for these objectives, and they reduced the multi-objective optimization and achievability (what they call Pareto realizability) problems for MDPs with discounted rewards to multi-objective linear programming. They were thus able to apply the results of [PY00] in order to approximate the Pareto curve for this problem. We work in an undiscounted setting, where objectives can be arbitrary $\omega$-regular properties. In our setting, strategies may require both randomization and memory in order to achieve a given value vector. As described earlier, our algorithms first reduce multi-objective $\omega$-regular problems to multi-objective reachability problems, and we then solve multi-objective reachability problems by reducing them to multi-objective LP. For multi-objective reachabilility, we show randomized memoryless strategies do suffice. Our LP methods for multi-objective reachability are closely related to the LP methods used in [CMH06] (and see also, e.g., [Put94], Theorem 6.9.1., where a related result about discounted MDPs is established). However, in order to establish the results in our undiscounted setting, even for reachability we have to overcome some new obstacles that do not arise in the discounted case. In particular, whereas the "discounted frequencies" used in [CMH06] are always well-defined finite values under all strategies, the analogous undiscounted frequencies or "expected number of visits" can in general be infinite for an arbitrary strategy. This forces us to preprocess the MDPs in such a way that ensures that a certain family of undiscounted stochastic flow equations has a finite solution which corresponds to the "expected number of visits" at each state-action pair under a given (memoryless) strategy. It also forces us to give a quite different proof that memoryless strategies suffice to achieve any achievable vector for multi-objective reachability, based on the convexity of the memorylessly achievable set.

Multi-objective MDPs have also been studied extensively in the OR and stochastic control literature (see e.g. [Fur80, Whi82, Hen83, Gho90, WT98]). Much of this work is typically concerned with discounted reward or long-run average reward models, and does not focus on the complexity of algorithms. None of this work seems to directly imply even our result that for multiple reachability objectives checking achievability of a value vector can be decided in polynomial time, not to mention the more general results for multi-objective model checking. 


\section{BASICS AND BACKGROUND}

A finite-state MDP $M=(V, \Gamma, \delta)$ consists of a finite set $V$ of states, an action alphabet $\Gamma$, and a transition relation $\delta$. Associated with each state $v$ is a set of enabled actions $\Gamma_{v} \subseteq \Gamma$. The transition relation is given by $\delta \subseteq V \times \Gamma \times[0,1] \times V$. For each state $v \in V$, each enabled action $\gamma \in \Gamma_{v}$, and every state $v^{\prime} \in V$, we have at most one transition $\left(v, \gamma, p_{\left(v, \gamma, v^{\prime}\right)}, v^{\prime}\right) \in \delta$, for some probability $p_{\left(v, \gamma, v^{\prime}\right)} \in(0,1]$, such that $\sum_{v^{\prime} \in V} p_{\left(v, \gamma, v^{\prime}\right)}=1$. When there is no transition $\left(v, \gamma, p_{\left(v, \gamma, v^{\prime}\right)}, v^{\prime}\right)$, we may, only for notational convenience, sometimes assume that there is a probability 0 transition, i.e., that $p_{\left(v, \gamma, v^{\prime}\right)}=0$. (But such redundant probability 0 transitions are not part of the actual input.) Thus, at each state, each enabled action determines a probability distribution on the next state. There are no other transitions, so no transitions on disabled actions. We assume every state $v$ has some enabled action, i.e., $\Gamma_{v} \neq \emptyset$, so there are no dead ends. For our complexity analysis, we assume of course that all probabilities $p_{\left(v, \gamma, v^{\prime}\right)}$ are rational. There are other ways to present MDPs, e.g., by separating controlled and probabilistic nodes into distinct states. The different presentations are equivalent and efficiently translatable to each other.

A labeled MDP $M=(V, \Gamma, \delta, l)$ has, in addition, a set of propositional predicates $Q=\left\{Q_{1}, \ldots, Q_{r}\right\}$ which label the states. We view this as being given by a labelling function $l: V \mapsto \Sigma$, where $\Sigma=2^{Q}$. We define the encoding size of a (labeled) MDP $M$, denoted by $|M|$, to be the total size required to encode all transitions and their rational probabilities, where rational values are encoded with numerator and denominator given in binary, as well as all state labels.

For a labeled $\operatorname{MDP} M=(V, \Gamma, \delta, l)$ with a given initial state $u \in V$, which we denote by $M_{u}$, runs of $M_{u}$ are infinite sequences of states $\pi=\pi_{0} \pi_{1} \ldots \in V^{\omega}$, where $\pi_{0}=u$ and for all $i \geq 0, \pi_{i} \in V$ and there is a transition $\left(\pi_{i}, \gamma, p, \pi_{i+1}\right) \in \delta$, for some $\gamma \in \Gamma_{\pi_{i}}$ and some probability $p>0$. Each run induces an $\omega$-word over $\Sigma$, namely $l(\pi) \doteq l\left(\pi_{0}\right) l\left(\pi_{1}\right) \ldots \in \Sigma^{\omega}$.

A strategy is a function $\sigma:(V \Gamma)^{*} V \mapsto \mathcal{D}(\Gamma)$, which maps a finite history of play to a probability distribution on the next action. Here $\mathcal{D}(\Gamma)$ denotes the set of probability distributions on the set $\Gamma$. Moreover, it must be the case that for all histories $w u, \sigma(w u) \in$ $\mathcal{D}\left(\Gamma_{u}\right)$, i.e., the probability distribution has support only over the actions available at state $u$. A strategy is pure if $\sigma(w u)$ has support on exactly one action, i.e., with probability 1 a single action is played at every history. A strategy is memoryless (stationary) if the strategy depends only on the last state, i.e., if $\sigma(w u)=\sigma\left(w^{\prime} u\right)$ for all $w, w^{\prime} \in(V \Gamma)^{*}$. If $\sigma$ is memoryless, we can simply define it as a function $\sigma: V \mapsto \mathcal{D}(\Gamma)$. An MDP $M$ with initial state $u$, together with a strategy $\sigma$, naturally induces a Markov chain $M_{u}^{\sigma}$, whose states are the histories of play in $M_{u}$, and such that from state $s=w v$ if $\gamma \in \Gamma_{v}$, there is a transition to state $s^{\prime}=w v \gamma v^{\prime}$ with probability $\sigma(w v)(\gamma) \cdot p_{\left(v, \gamma, v^{\prime}\right)}$. A run $\theta$ in $M_{u}^{\sigma}$ is thus given by a sequence $\theta=\theta_{0} \theta_{1} \ldots$, where $\theta_{0}=u$ and each $\theta_{i} \in(V \Gamma)^{*} V$, for all $i \geq 0$. We associate to each history $\theta_{i}=w v$ the label of its last state $v$. In other words, we overload the notation and define $l(w v) \doteq l(v)$. We likewise associate with each run $\theta$ the $\omega$-word $l(\theta) \doteq l\left(\theta_{0}\right) l\left(\theta_{1}\right) \ldots$ Suppose we are given $\varphi$, an LTL formula or Büchi automaton, or any other formalism for expressing an $\omega$-regular language over alphabet $\Sigma$. Let $L(\varphi) \subseteq \Sigma^{\omega}$ denote the language expressed by $\varphi$. We write $\operatorname{Pr}_{u}^{\sigma}(\varphi)$ to denote the probability that a trajectory $\theta$ of $M_{u}^{\sigma}$ satistifies $\varphi$, i.e., that $l(\theta) \in L(\varphi)$. For generality, rather than just allowing an initial vertex $u$ we allow an initial probability distribution $\alpha \in \mathcal{D}(V)$. Let $\operatorname{Pr}_{\alpha}^{\sigma}(\varphi)$ denote the probability that under strategy $\sigma$, starting with initial distribution $\alpha$, 
we will satisfy $\omega$-regular property $\varphi$. These probabilities are well defined because the set of such runs is Borel measurable (see, e.g., [Var85, CY95]).

As in the introduction, for a $k$-tuple of $\omega$-regular properties $\Phi=\left\langle\varphi_{1}, \ldots, \varphi_{k}\right\rangle$, given a strategy $\sigma$, we let $t^{\sigma}=\left(t_{1}^{\sigma}, \ldots, t_{k}^{\sigma}\right)$, with $t_{i}^{\sigma}=\operatorname{Pr}_{u}^{\sigma}\left(\varphi_{i}\right)$, for $i=1, \ldots, k$. For MDP $M$ and starting state $u$, we define the achievable set of value vectors with respect to $\Phi$ to be $U_{M_{u}, \Phi}=\left\{r \in \mathbb{R}_{>0}^{k} \mid \exists \sigma\right.$ such that $\left.t^{\sigma} \geq r\right\}$. For a set $U \subseteq \mathbb{R}^{k}$, we define a subset $\mathcal{P} \subseteq U$ of it, called the Pareto curve or the Pareto set of $U$, consisting of the set of Pareto optimal (or Pareto efficient) vectors inside $U$. A vector $v \in U$ is called Pareto optimal if $\neg \exists v^{\prime}\left(v^{\prime} \in U \wedge v \leq v^{\prime} \wedge v \neq v^{\prime}\right)$. Thus $\mathcal{P}=\{v \in U \mid v$ is Pareto optimal $\}$. We use $\mathcal{P}_{M_{u}, \Phi} \subseteq U_{M_{u}, \Phi}$ to denote the Pareto curve of $U_{M_{u}, \Phi}$.

It is clear, e.g., from Figure1, that the Pareto curve is in general an infinite set. In fact, it follows from our results that for general $\omega$-regular objectives the Pareto set is a convex polyhedral set. In principle, we may want to compute some kind of exact representation of this set by, e.g., enumerating all the vertices (on the upper envelope) of the polytope that defines the Pareto curve, or enumerating the facets that define it. It is not possible to do this in polynomial-time in general. In fact, the following theorem holds:

Theorem 2.1. There is a family of $M D P s,\langle M(n) \mid n \in \mathbb{N}\rangle$, where $M(n)$ has $n$ states and size $O(n)$, such that for $M(n)$ the Pareto curve for two reachability objectives, $\diamond P_{1}$ and $\diamond P_{2}$, contains $n^{\Omega(\log n)}$ vertices (and thus $n^{\Omega(\log n)}$ facets).

Proof. We will adapt and build on a known construction for the bi-objective shortest path problem which shows that the Pareto curve for that problem can have $n^{\Omega(\log n)}$ vertices. This was shown in Car83 and a simplified proof (using a similar construction) was given in MS01. (The constructions and theorems there are phrased in terms of parametric shortest paths, but these are equivalent to bi-objective shortest paths.) What those constructions show is that, for some polynomial $f$, and for every $n$, there is a graph $G_{n}$ with $f(n)$ nodes and distinguished nodes $s$ and $t$, and such that every edge $(u, v)$ has two (positive) costs $c(u, v)$ and $d(u, v)$, which yield two cost functions $c(\cdot)$ and $d(\cdot)$ on the $s$-t paths, such that the Pareto curve of the $s$ - $t$ paths under the two objectives has $n^{\Omega(\log n)}$ vertices (and edges). An important property of the constructed graphs $G_{n}$ is that they are acyclic and layered, that is, the nodes are arranged in layers $L_{0}=s, L_{1}, L_{2}, \ldots, L_{n}=t$, and all edges are from layer $L_{i}$ to $L_{i+1}$ for some $i \in\{0, \ldots, n-1\}$.

Building on this construction, we now construct the following instance $M_{n}$ of the MDP problem with two reachability objectives. The states of $M_{n}$ are the same as $G_{n}$ with 2 extra absorbing states: the red state $R$, and the blue state $B$, which are the two target states of our two reachability objectives. For each state $u$ there is one action for each outgoing edge $(u, v)$; if we choose this action then we transition with probability $r(u, v)$ to state $R$, with probability $b(u, v)$ to $B$, with probability $1 / 2$ to $v$, and with the remaining probability to $t$. The probabilities $r(u, v)$ and $b(u, v)$ are defined as follows. Let $h$ be the maximum $c$ or $d$ cost over all the edges. For an edge $(u, v)$ where $u \in L_{i}$ (and $v \in L_{i+1}$ ), set

$$
r(u, v):=\frac{2^{i}(2 h-c(u, v))}{8 h 2^{n}}
$$

and

$$
b(u, v):=\frac{2^{i}(2 h-d(u, v))}{8 h 2^{n}}
$$

Note that both these quantities are in the interval $[0,1 / 4]$, so all probabilities are welldefined. 
The claim is that there is a 1-1 correspondence between the vertices of the Pareto curve of this MDP $M_{n}$ and the Pareto curve of the bi-objective shortest path on $G_{n}$. First we note that the vertices of the Pareto curve for the MDP correspond to pure memoryless strategies (meaning that for each vertex of the Pareto curve a pure memoryless strategy can achieve the value vector that the vertex defines). The reason for this is that the vertices are optima for a linear combination of the two objectives, and it follows from the proof of Theorem 3.2, which we shall show later, that these objectives have pure memoryless optimal strategies.

A pure strategy corresponds to a path from $s$ to $t$. Let $\pi=s, u_{1}, u_{2}, \ldots, u_{n-1} t$ be such a path/strategy. The probability that this strategy leads to the red node $R$ is $r\left(s, u_{1}\right)+\ldots+$ $\operatorname{Prob}\left(\right.$ reach node $\left.u_{i}\right) * r\left(u_{i}, u_{i}+1\right)+\ldots$ The probability that the process reaches node $u_{i}$ under the strategy $\pi$ is $1 / 2^{i}$, independent of the path. Thus, $\operatorname{Prob}_{\pi}($ reach $R)=a-b * c(\pi)$, where $a, b$ are constants independent of the path. Similarly, $\operatorname{Prob}_{\pi}($ reach $B)=a-b * d(\pi)$.

It follows that minimizing the $c$ and $d$ costs of the paths is equivalent to maximizing the probabilities of reaching $R$ and $B$, and this also holds for any positive linear combination of the two respective objectives. Thus, there is a correspondence between their Pareto curves.

So, the Pareto curve is in general a polyhedral surface of superpolynomial size, and thus cannot be constructed exactly in polynomial time. We show, however, that the Pareto set can be efficiently approximated to any desired accuracy $\epsilon>0$. An $\epsilon$-approximate Pareto curve, $\mathcal{P}_{M_{u}, \Phi}(\epsilon) \subseteq U_{M_{u}, \Phi}$, is any achievable set such that $\forall r \in U_{M_{u}, \Phi} \exists t \in \mathcal{P}_{M_{u}, \Phi}(\epsilon)$ such that $r \leq(1+\epsilon) t$. When the subscripts $M_{u}$ and $\Phi$ are clear from the context, we will drop them and use $U, \mathcal{P}$, and $\mathcal{P}(\epsilon)$ to denote the achievable set, Pareto set, and $\epsilon$-approximate Pareto set, respectively.

We also consider general multi-objective queries. A quantitative predicate over $\omega$-regular property $\varphi_{i}$ is a statement of the form $\operatorname{Pr}_{u}^{\sigma}\left(\varphi_{i}\right) \Delta p$, for some rational probability $p \in[0,1]$, and where $\Delta$ is a comparison operator $\Delta \in\{\leq, \geq,<,>,=\}$. Suppose $B$ is a boolean combination over such predicates. Then, given $M$ and $u$, and $B$, we can ask whether there exists a strategy $\sigma$ such that $B$ holds, or whether $B$ holds for all $\sigma$. Note that since $B$ can be put in DNF form, and the quantification over strategies pushed into the disjunction, and since $\omega$-regular languages are closed under complementation, any query of the form $\exists \sigma B$ (or of the form $\forall \sigma B$ ) can be transformed to a disjunction (a negated disjunction, respectively) of queries of the form:

$$
\exists \sigma \bigwedge_{i}\left(\operatorname{Pr}_{u}^{\sigma}\left(\varphi_{i}\right) \geq r_{i}\right) \wedge \bigwedge_{j}\left(\operatorname{Pr}_{u}^{\sigma}\left(\psi_{j}\right)>r_{j}^{\prime}\right)
$$

We call queries of the form (1) extended achievability queries. Thus, if the multiobjective query is fixed, it suffices to perform a fixed number of extended achievability queries to decide any multi-objective query. Note, however, that the number of extended achievability queries we need could be exponential in the size of $B$. We do not focus on optimizing query complexity in this paper.

A motivation for allowing general multi-objective queries is to enable assume-guarantee compositional reasoning for probabilistic systems. Consider, e.g., a probabilistic system consisting of the concurrent composition of two components, $M_{1}$ and $M_{2}$, where output from $M_{1}$ provides input to $M_{2}$ and thus controls $M_{2}$. We denote this by $M_{1} \triangleright M_{2}$. $M_{2}$ itself may generate outputs for some external device, and $M_{1}$ may also be controlled by external inputs. (One can also consider symmetric composition, where outputs from both 


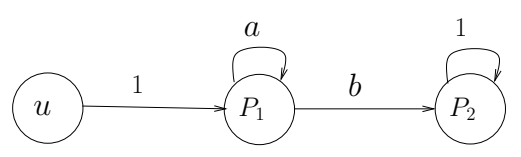

Figure 2: The MDP $M^{\prime}$.

components provide inputs to both. Here, for simplicity, we restrict ourselves to asymmetric composition where $M_{1}$ controls $M_{2}$.) Let $M$ be an MDP with separate input and output action alphabets $\Sigma_{1}$ and $\Sigma_{2}$, and let $\varphi_{1}$ and $\varphi_{2}$ denote $\omega$-regular properties over these two alphabets, respectively. We write $\left\langle\varphi_{1}\right\rangle_{\geq r_{1}} M\left\langle\varphi_{2}\right\rangle_{\geq r_{2}}$, to denote the assertion that "if the input controller of $M$ satisfies $\varphi_{1}$ with probability $\geq r_{1}$, then the output generated by $M$ satisfies $\varphi_{2}$ with probability $\geq r_{2}$ ". Using this, we can formulate a general compositional assume-guarantee proof rule:

$$
\begin{gathered}
\left\langle\varphi_{1}\right\rangle \geq r_{1} M_{1}\left\langle\varphi_{2}\right\rangle_{\geq r_{2}} \\
\left\langle\varphi_{2}\right\rangle_{\geq r_{2}} M_{2}\left\langle\varphi_{3}\right\rangle_{\geq r_{3}} \\
\left\langle\varphi_{1}\right\rangle_{\geq r_{1}} M_{1} \triangleright M_{2}\left\langle\varphi_{3}\right\rangle \geq r_{3}
\end{gathered}
$$

Thus, to check $\left\langle\varphi_{1}\right\rangle_{\geq r_{1}} M_{1} \triangleright M_{2}\left\langle\varphi_{3}\right\rangle_{\geq r_{3}}$ it suffices to check two properties of smaller systems: $\left\langle\varphi_{1}\right\rangle_{\geq r_{1}} M_{1}\left\langle\varphi_{2}\right\rangle_{\geq r_{2}}$ and $\left\langle\varphi_{2}\right\rangle_{\geq r_{2}} M_{2}\left\langle\varphi_{3}\right\rangle_{\geq r_{3}}$. Note that checking $\left\langle\varphi_{1}\right\rangle_{\geq r_{1}} M\left\langle\varphi_{2}\right\rangle_{\geq r_{2}}$ amounts to checking that there does not exist a strategy $\sigma$ controlling $M$ such that $\operatorname{Pr}_{u}^{\sigma}\left(\varphi_{1}\right) \geq r_{1}$ and $\operatorname{Pr}_{u}^{\sigma}\left(\varphi_{2}\right)<r_{2}$.

We also consider qualitative multi-objective queries. These are queries restricted so that $B$ contains only qualitative predicates of the form $\operatorname{Pr}_{u}^{\sigma}\left(\varphi_{i}\right) \Delta b$, where $b \in\{0,1\}$. These can, e.g., be used to check qualitative assume-guarantee conditions of the form: $\left\langle\varphi_{1}\right\rangle_{\geq 1} M\left\langle\varphi_{2}\right\rangle_{\geq 1}$. It is not hard to see that again, via boolean manipulations and complementation of automata, we can convert any qualitative query to a number of queries of the form:

$$
\exists \sigma \bigwedge_{\varphi \in \Phi}\left(\operatorname{Pr}_{u}^{\sigma}(\varphi)=1\right) \wedge \bigwedge_{\psi \in \Psi}\left(\operatorname{Pr}_{u}^{\sigma}(\psi)>0\right)
$$

where $\Phi$ and $\Psi$ are sets of $\omega$-regular properties. It thus suffices to consider only these qualitative queries.

In the next sections we study how to decide various classes of multi-objective queries, and how to approximate the Pareto curve for properties $\Phi$. Let us observe here a difficulty that we will have to deal with. Namely, in general we will need both randomization and memory in our strategies in order to satisfy even simple qualitative multi-objective queries. Consider the MDP, $M^{\prime}$, shown in Figure 2, and consider the conjunctive query: $B \equiv$ $\operatorname{Pr}_{u}^{\sigma}\left(\square \diamond P_{1}\right)>0 \wedge \operatorname{Pr}_{u}^{\sigma}\left(\square \diamond P_{2}\right)>0$. It is not hard to see that starting at state $u$ in $M^{\prime}$ any strategy $\sigma$ that satisfies $B$ must use both memory and randomization. Each predicate in $B$ can be satisfied in isolation (in fact with probability 1 ), but, with a memoryless or deterministic strategy, if we try to satisfy $\square \diamond P_{2}$ with non-zero probability, we will be forced to satisfy $\square \diamond P_{1}$ with probability 0 . Note, however, that we can satisfy both with probability $>0$ using a strategy that uses both memory and randomness: namely, upon reaching the state labeled $P_{1}$ for the first time, with probability $1 / 2$ we use move $a$ and with probability $1 / 2$ we use move $b$. Thereafter, upon encountering the state labeled $P_{1}$ for the $n$th time, $n \geq 2$, we deterministically pick action $a$. This clearly assures that both predicates are satisfied with probability $=1 / 2>0$. 
We note that our results (combined with the earlier results of [CY98]) imply that for general multi-objective queries a randomized strategy with a finite amount of memory (which depends on the MDP and query) does suffice to satisfy any satisfiable quantitative multi-objective $\omega$-regular query.

\section{Multi-objective Reachability}

In this section, as a step towards quantitative multi-objective model checking problems, we study a simpler multi-objective reachability problem. Specifically, we are given an MDP, $M=(V, \Gamma, \delta)$, a starting state $u$, and a collection of target sets $F_{i} \subseteq V, i=1, \ldots, k$. The sets $F_{i}$ may overlap. We have $k$ objectives: the $i$-th objective is to maximize the probability of $\diamond F_{i}$, i.e., of reaching some state in $F_{i}$. We assume that the states $F=\bigcup_{i=1}^{k} F_{i}$ are all absorbing states with a self-loop. In other words, for all $v \in F,(v, a, 1, v) \in \delta$ and $\Gamma_{v}=\{a\}$. (The assumption that target states are absorbing is necessary for the proofs in this section, but it is not a restriction in general for our results. It will follow from the model checking results in Section 5, which build on this section, that multi-objective reachability problems for arbitrary target states (whether absorbing or not) can also be handled with the same complexities.)

We first need to do some preprocessing on the MDP, to remove some useless states. For each state $v \in V \backslash F$ we can check easily whether there exists a strategy $\sigma$ such that $\operatorname{Pr}_{v}^{\sigma}(\diamond F)>0$ : this just amounts to checking whether there exists a path from $v$ to $F$ in the underlying graph of the MDP, i.e., the graph given by considering only the non-zeroprobability transitions. Let us call a state that does not satisfy this property a bad state. Clearly, for the purposes of optimizing reachability objectives, we can compute and remove all bad states from an MDP. Thus, it is safe to assume that bad states do not exist 1 Let us call an MDP with goal states $F$ cleaned-up if it does not contain any bad states.

Proposition 3.1. For a cleaned-up $M D P$, an initial distribution $\alpha \in \mathcal{D}(V \backslash F)$, and a vector of probabilities $r \in[0,1]^{k}$, there exists a (memoryless) strategy $\sigma$ such that

$$
\bigwedge_{i=1}^{k} \operatorname{Pr}_{\alpha}^{\sigma}\left(\diamond F_{i}\right) \geq r_{i}
$$

if and only if there exists a (respectively, memoryless) strategy $\sigma^{\prime}$ such that

$$
\bigwedge_{i=1}^{k} \operatorname{Pr}_{\alpha}^{\sigma^{\prime}}\left(\diamond F_{i}\right) \geq r_{i} \wedge \bigwedge_{v \in V} \operatorname{Pr}_{v}^{\sigma^{\prime}}(\diamond F)>0 .
$$

Proof. This is quite obvious, but we give a quick argument anyway. Suppose we have such a strategy $\sigma$. Since the MDP is cleaned-up, we know that from every state in $V$ we can reach $F$ with a positive probability. Suppose the strategy leads to a history whose last state is $v \in V \backslash F$, and that thereafter the strategy is such that it will never reach $F$ on any path. We simply revise $\sigma$ to a strategy $\sigma^{\prime}$ such that, if we ever arrive at such a "dead" history, we

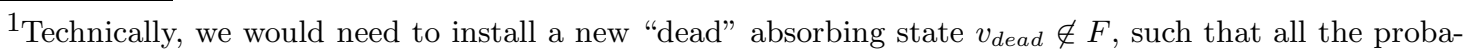
bilities going into states that have been removed now go to $v_{\text {dead }}$. For convenience in notation, instead of explicitly adding $v_{\text {dead }}$ we treat it as implicit: we allow that for some states $v \in V$ and some action $a \in \Gamma_{v}$ we have $\sum_{v^{\prime} \in V} p_{\left(v, \gamma, v^{\prime}\right)}<1$, and we implicitly assume that there is an "invisible" transition to $v_{\text {dead }}$ with the residual probability, i.e., with $p_{\left(v, \gamma, v_{\text {dead }}\right)}=1-\sum_{v^{\prime} \in V} p_{\left(v, \gamma, v^{\prime}\right)}$. Of course, $v_{\text {dead }}$ would then be a "bad" state, but we can ignore this implicit state.
} 
Objectives $(i=1, \ldots, k): \quad$ Maximize $\sum_{v \in F_{i}} y_{v}$

\section{Subject to:}

$$
\begin{array}{lll}
\sum_{\gamma \in \Gamma_{v}} y_{(v, \gamma)}-\sum_{v^{\prime} \in V} \sum_{\gamma^{\prime} \in \Gamma_{v^{\prime}}} p_{\left(v^{\prime}, \gamma^{\prime}, v\right)} y_{\left(v^{\prime}, \gamma^{\prime}\right)}=\alpha(v) & & \text { for all } v \in V \backslash F ; \\
y_{v}-\sum_{v^{\prime} \in V \backslash F} \sum_{\gamma^{\prime} \in \Gamma_{v^{\prime}}} p_{\left(v^{\prime}, \gamma^{\prime}, v\right)} y_{\left(v^{\prime}, \gamma^{\prime}\right)} & =0 & \text { for all } v \in F ; \\
y_{v} & \geq 0 & \text { for all } v \in F ; \\
y_{(v, \gamma)} & \geq 0 & \text { for all } v \in V \backslash F \text { and } \gamma \in \Gamma_{u} .
\end{array}
$$

Figure 3: Multi-objective LP for the multi-objective MDP reachability problem

switch and play according to the memoryless strategy starting at $v$ which reaches $F$ with some positive probability. Note that if $\sigma$ is memoryless then so is $\sigma^{\prime}$.

Now, consider the multi-objective LP described in Figure 32 The set of variables in this LP are as follows: for each $v \in F$, there is a variable $y_{v}$, and for each $v \in V \backslash F$ and each $\gamma \in \Gamma_{v}$ there is a variable $y_{(v, \gamma)}$.

Theorem 3.2. Suppose we are given a cleaned-up $M D P, M=(V, \Gamma, \delta)$, with multiple target sets $F_{i} \subseteq V, i=1, \ldots, k$, where every target $v \in F=\bigcup_{i=1}^{k} F_{i}$ is an absorbing state. Let $\alpha \in \mathcal{D}(V \backslash F)$ be an initial distribution (in particular $V \backslash F \neq \emptyset$ ). Let $r \in(0,1]^{k}$ be a vector of positive probabilities. Then the following are all equivalent:

(1.) There is a (possibly randomized) memoryless strategy $\sigma$ such that

$$
\bigwedge_{i=1}^{k}\left(\operatorname{Pr}_{\alpha}^{\sigma}\left(\diamond F_{i}\right) \geq r_{i}\right)
$$

(2.) There is a feasible solution $y^{\prime}$ for the multi-objective LP in Fig. 3 such that

$$
\bigwedge_{i=1}^{k}\left(\sum_{v \in F_{i}} y_{v}^{\prime} \geq r_{i}\right)
$$

(3.) There is an arbitrary strategy $\sigma$ such that

$$
\bigwedge_{i=1}^{k}\left(\operatorname{Pr}_{\alpha}^{\sigma}\left(\diamond F_{i}\right) \geq r_{i}\right)
$$

Proof.

$(1.) \Rightarrow$ (2.). Since the MDP is cleaned up, by Proposition 3.1 we can assume there is a memoryless strategy $\sigma$ such that $\bigwedge_{i=1}^{k} \operatorname{Pr}_{\alpha}^{\sigma}\left(\diamond F_{i}\right) \geq r_{i}$ and $\forall v \in V \operatorname{Pr}_{v}^{\sigma}(\diamond F)>0$. Consider the square matrix $P^{\sigma}$ whose size is $|V \backslash F| \times|V \backslash F|$, and whose rows and columns are indexed by states in $V \backslash F$. The $\left(v, v^{\prime}\right)^{\prime}$ th entry of $P^{\sigma}, P_{v, v^{\prime}}^{\sigma}$, is the probability that starting in state $v$ we shall in one step end up in state $v^{\prime}$. In other words, $P_{v, v^{\prime}}^{\sigma}=\sum_{\gamma \in \Gamma_{v}} \sigma(v)(\gamma) \cdot p_{v, \gamma, v^{\prime}}$.

For all $v \in V \backslash F$, let $y_{(v, \gamma)}^{\prime}=\sum_{v^{\prime} \in V \backslash F} \alpha\left(v^{\prime}\right) \sum_{n=0}^{\infty}\left(P^{\sigma}\right)_{v^{\prime}, v}^{n} \sigma(v)(\gamma)$. In other words $y_{(v, \gamma)}^{\prime}$ denotes the "expected number of times that, using the strategy $\sigma$, starting in the distribution $\alpha$, we will visit the state $v$ and upon doing so choose action $\gamma$ ". We don't know yet that these

\footnotetext{
${ }^{2}$ We mention without further elaboration that this LP can be derived, using complementary slackness, from the dual LP of the standard LP for single-objective reachability obtained from Bellman's optimality equations, whose variables are $x_{v}$, for $v \in V$, and whose unique optimal solution is the vector $x^{*}$ with $x_{v}^{*}=\max _{\sigma} \operatorname{Pr}_{v}^{\sigma}(\diamond F)$ (see, e.g., Put94, CY98).
} 
are finite values, but assuming they are, for $v \in F$, let $y_{v}^{\prime}=\sum_{v^{\prime} \in V \backslash F} \sum_{\gamma^{\prime} \in \Gamma_{v^{\prime}}} p_{\left(v^{\prime}, \gamma^{\prime}, v\right)} y_{\left(v^{\prime}, \gamma^{\prime}\right)}^{\prime}$. This completes the definition of the entire vector $y^{\prime}$.

Lemma 3.3. The vector $y^{\prime}$ is well defined (i.e., all entries $y_{(v, \gamma)}^{\prime}$ are finite). Moreover, $y^{\prime}$ is a feasible solution to the constraints of the LP in Figure 3 .

Proof. First, we show that for all $v \in V \backslash F$ and $\gamma \in \Gamma_{v}, y_{(v, \gamma)}^{\prime}$ is a well defined finite value. It then also follows from the definition of $y_{v}^{\prime}$ that $y_{v}^{\prime}$ is also finite and thus that the vector $y^{\prime}$ is well defined. Note that because $\sigma$ has the property that $\forall v \in V \quad \operatorname{Pr}_{v}^{\sigma}(\diamond F)>0, P^{\sigma}$ is clearly a substochastic matrix with the property that, for some power $d \geq 1$, all of the row sums of $\left(P^{\sigma}\right)^{d}$ are strictly less than 1 . Thus, it follows that $\lim _{n \rightarrow \infty}\left(P^{\sigma}\right)^{n} \rightarrow \mathbf{0}$, and thus by standard facts about matrices the inverse matrix $\left(I-P^{\sigma}\right)^{-1}=\sum_{n=0}^{\infty}\left(P^{\sigma}\right)^{n}$ exists and is non-negative. Now observe that

$$
\begin{aligned}
y_{(v, \gamma)}^{\prime} & =\sum_{v^{\prime} \in V \backslash F} \alpha\left(v^{\prime}\right) \sum_{n=0}^{\infty}\left(P^{\sigma}\right)_{v^{\prime}, v}^{n} \sigma(v)(\gamma) \\
& =\sum_{v^{\prime} \in V \backslash F} \alpha\left(v^{\prime}\right) \sigma(v)(\gamma) \sum_{n=0}^{\infty}\left(P^{\sigma}\right)_{v^{\prime}, v}^{n} \\
& =\sigma(v)(\gamma) \sum_{v^{\prime} \in V \backslash F} \alpha\left(v^{\prime}\right)\left(I-P^{\sigma}\right)_{v^{\prime}, v}^{-1}
\end{aligned}
$$

Next, we show that $y^{\prime}$ is a feasible solution to the constraints in the multi-objective LP in Figure 3. Note that, for each state $v \in V \backslash F$, the expression $\sum_{v^{\prime} \in V} \sum_{\gamma^{\prime} \in \Gamma_{v^{\prime}}} p_{\left(v^{\prime}, \gamma^{\prime}, v\right)} y_{\left(v^{\prime}, \gamma^{\prime}\right)}^{\prime}$ is precisely the "expected number of times we will take a transition into the state $v$ " if we start at initial distribution $\alpha$ and using strategy $\sigma$, whereas $\sum_{\gamma \in \Gamma_{v}} y_{(v, \gamma)}^{\prime}$ defines precisely the "expected number of times we will take a transition out of the state $v$ ". Thus $\alpha(v)$, the probability that we will start in state $v$, is precisely given by $\sum_{\gamma \in \Gamma_{v}} y_{(v, \gamma)}^{\prime}-$ $\sum_{v^{\prime} \in V} \sum_{\gamma^{\prime} \in \Gamma_{v^{\prime}}} p_{\left(v^{\prime}, \gamma^{\prime}, v\right)} y_{\left(v^{\prime}, \gamma^{\prime}\right)}^{\prime}=\alpha(v)$. More formally, for each state $v \in V \backslash F$ :

$$
\begin{aligned}
\sum_{v^{\prime} \in V} \sum_{\gamma^{\prime} \in \Gamma_{v^{\prime}}} p_{\left(v^{\prime}, \gamma^{\prime}, v\right)} y_{\left(v^{\prime}, \gamma^{\prime}\right)}^{\prime} & =\sum_{v^{\prime} \in V} \sum_{\gamma^{\prime} \in \Gamma_{v^{\prime}}} p_{\left(v^{\prime}, \gamma^{\prime}, v\right)} \sum_{v^{\prime \prime} \in V \backslash F} \alpha\left(v^{\prime \prime}\right) \sum_{n=0}^{\infty}\left(P^{\sigma}\right)_{v^{\prime \prime}, v^{\prime}}^{n} \sigma\left(v^{\prime}\right)\left(\gamma^{\prime}\right) \\
& =\sum_{v^{\prime \prime} \in V \backslash F} \alpha\left(v^{\prime \prime}\right) \sum_{v^{\prime} \in V} \sum_{\gamma^{\prime} \in \Gamma_{v^{\prime}}} p_{\left(v^{\prime}, \gamma^{\prime}, v\right)} \sum_{n=0}^{\infty}\left(P^{\sigma}\right)_{v^{\prime \prime}, v^{\prime}}^{n} \sigma\left(v^{\prime}\right)\left(\gamma^{\prime}\right) \\
& =\sum_{v^{\prime \prime} \in V \backslash F} \alpha\left(v^{\prime \prime}\right) \sum_{n=1}^{\infty}\left(P^{\sigma}\right)_{v^{\prime \prime}, v}^{n}
\end{aligned}
$$

The last expression is easily seen to be the expected number of times we will transition into state $v$. It is clear by linearity of expectations that $\sum_{\gamma \in \Gamma_{v}} y_{(v, \gamma)}^{\prime}$ gives the expected number of times we will transition out of state $v$. It is thus clear that $\sum_{\gamma \in \Gamma_{v}} y_{(v, \gamma)}^{\prime}-$ $\sum_{v^{\prime} \in V} \sum_{\gamma^{\prime} \in \Gamma_{v^{\prime}}} p_{\left(v^{\prime}, \gamma^{\prime}, v\right)} y_{\left(v^{\prime}, \gamma^{\prime}\right)}^{\prime}=\alpha(v)$.

Now we argue that $\sum_{v \in F_{i}} y_{v}^{\prime}=\operatorname{Pr}_{\alpha}^{\sigma}\left(\diamond F_{i}\right)$. To see this, note that for $v \in F, y_{v}^{\prime}=$ $\sum_{v^{\prime} \in V \backslash F} \sum_{\gamma^{\prime} \in \Gamma_{v^{\prime}}} p_{\left(v^{\prime}, \gamma^{\prime}, v\right)} y_{\left(v^{\prime}, \gamma^{\prime}\right)}^{\prime}$ is precisely the "expected number of times that we will transition into state $v$ for the first time", starting at distribution $\alpha$. The reason we can say "for 
the first time" is because only the states in $V \backslash F$ are included in the matrix $P^{\sigma}$. But note that this italicised statement in quotes is another way to define the probability of eventually reaching state $v$. This equality can be establish formally, but we omit the formal algebraic derivation here. Thus $\sum_{v \in F_{i}} y_{v}^{\prime}=\operatorname{Pr}_{\alpha}^{\sigma}\left(\diamond F_{i}\right) \geq r_{i}$. We are done with (1.) $\Rightarrow$ (2.).

(2.) $\Rightarrow$ (1.). We now wish to show that if $y^{\prime \prime}$ is a feasible solution to the multi-objective LP such that $\sum_{v \in F_{i}} y_{v}^{\prime \prime} \geq r_{i}>0$, for all $i=1, \ldots, k$, then there exists a memoryless strategy $\sigma$ such that $\bigwedge_{i=1}^{k} \operatorname{Pr}_{\alpha}^{\sigma}\left(\diamond F_{i}\right) \geq r_{i}$.

Suppose we have such a solution $y^{\prime \prime}$. Let $S=\left\{v \in V \backslash F \mid \sum_{\gamma \in \Gamma_{v}} y_{(v, \gamma)}^{\prime \prime}>0\right\}$. Let $\sigma$ be the memoryless strategy, given as follows. For each $v \in S$

$$
\sigma(v)(\gamma):=\frac{y_{(v, \gamma)}^{\prime \prime}}{\sum_{\gamma^{\prime} \in \Gamma_{v}} y_{v, \gamma^{\prime}}^{\prime \prime}}
$$

Note that since $\sum_{\gamma \in \Gamma_{v}} y_{(v, \gamma)}^{\prime \prime}>0, \sigma(v)$ is a well-defined probability distribution on the moves at state $v \in S$. For the remaining states $v \in(V \backslash F) \backslash S$, let $\sigma(v)$ be an arbitrary distribution in $\mathcal{D}\left(\Gamma_{v}\right)$.

Lemma 3.4. This memoryless strategy $\sigma$ satisfies $\bigwedge_{i=1}^{k} \operatorname{Pr}_{\alpha}^{\sigma}\left(\diamond F_{i}\right) \geq r_{i}$.

Proof. Let us assume, for the sake of convenience in our analysis, that there is an extra dead-end absorbing state $v_{\text {dead }} \notin F$ available, and an extra move $\gamma_{\text {dead }}$ available at each state, $v$, with $p_{\left(v, \gamma_{\text {dead }}, v_{\text {dead }}\right)}=1$, and for each $v \in(V \backslash F) \backslash S$, instead of letting $\sigma(v)$ be arbitrary, let $\sigma(v)\left(\gamma_{\text {dead }}\right)=1$. In other words, from each such state we simply move directly to an absorbing dead-end which is outside of $F$. The assumption that such a dead-end exists is just for convenience: clearly, without such a dead-end, we can use any (mixed) move at such vertices in our strategy, and such a strategy would yield at least as high a value for $\operatorname{Pr}_{\alpha}^{\sigma}\left(\diamond F_{i}\right)$, for all $i=1, \ldots, k$.

Let us now explain the reason why we don't care about what moves are used at states outside $S$ in the strategy $\sigma$. Let support $(\alpha)=\{v \in V \backslash F \mid \alpha(v)>0\}$. We claim $S$ contains all states reachable from support $(\alpha)$ using strategy $\sigma$. To see this, first note that support $(\alpha) \subseteq S$, because for all $v \in \operatorname{support}(\alpha)$, since $\sum_{\gamma \in \Gamma_{v}} y_{(v, \gamma)}^{\prime \prime}-$ $\sum_{v^{\prime} \in V} \sum_{\gamma^{\prime} \in \Gamma_{v^{\prime}}} p_{\left(v^{\prime}, \gamma^{\prime}, v\right)} y_{\left(v^{\prime}, \gamma^{\prime}\right)}^{\prime \prime}=\alpha(v)$ and $\alpha(v)>0$, and since $y_{v^{\prime}, \gamma^{\prime}}^{\prime \prime} \geq 0$ for all $v^{\prime} \in V \backslash F$ and $\gamma^{\prime} \in \Gamma_{v^{\prime}}$, it must be the case that, $\sum_{\gamma \in \Gamma_{v}} y_{(v, \gamma)}^{\prime \prime}>0$. Thus support $(\alpha) \subseteq S$. Inductively, for $k \geq 0$, consider any state $v \in V \backslash F$, such that we can, with non-zero probability, reach $v$ in $k$ steps using strategy $\sigma$ from a state in support $(\alpha)$, and such that we can not reach $v$ (with non-zero probability) in any fewer than $k$ step. For the base case $k=0$, we already know $v \in \operatorname{support}(\alpha) \subseteq S$. For $k>0$, we must have $\alpha(v)=0$. But note that there must be a positive probability of moving to $v$ in one step from some other state $v^{\prime}$ which can be reached in $k-1$ steps from support $(\alpha)$. But this is so if and only if for some $\gamma^{\prime} \in \Gamma_{v^{\prime}}$, both $p_{\left(v^{\prime}, \gamma^{\prime}, v\right)}>0$ and $y_{\left(v^{\prime}, \gamma^{\prime}\right)}^{\prime \prime}>0\left(\right.$ and thus $\left.\sigma\left(v^{\prime}\right)\left(\gamma^{\prime}\right)>0\right)$. Hence, $\sum_{v^{\prime} \in V} \sum_{\gamma^{\prime} \in \Gamma_{v^{\prime}}} p_{\left(v^{\prime}, \gamma^{\prime}, v\right)} y_{\left(v^{\prime}, \gamma^{\prime}\right)}^{\prime \prime}>0$. Thus since $\sum_{\gamma \in \Gamma_{v}} y_{(v, \gamma)}^{\prime \prime}-\sum_{v^{\prime} \in V} \sum_{\gamma^{\prime} \in \Gamma_{v^{\prime}}} p_{\left(v^{\prime}, \gamma^{\prime}, v\right)} y_{\left(v^{\prime}, \gamma^{\prime}\right)}^{\prime \prime}=0$, we must have $\sum_{\gamma \in \Gamma_{v}} y_{(v, \gamma)}^{\prime \prime}>0$, and thus $v \in S$. Hence $S$ contains the set of nodes reachable from nodes in the support of the initial distribution, support $(\alpha)$, using the strategy $\sigma$.

We will now show that $\operatorname{Pr}_{\alpha}^{\sigma}\left(\diamond F_{i}\right) \geq r_{i}$, for all $i=1, \ldots, k$. Let us consider the underlying graph of the "flows" defined by $y^{\prime \prime}$. Namely, let $G=(V, E)$ be a graph on states of $M$ such that $\left(v, v^{\prime}\right) \in E$ if and only if there is some $\gamma \in \Gamma_{v}$ such that $y_{(v, \gamma)}^{\prime \prime}>0$ and $p_{\left(v, \gamma, v^{\prime}\right)}>0$. Let 
$W \subseteq V \backslash F$ be the set of vertices in $V \backslash F$ that have a non-zero "flow" to $F$, i.e., $v$ is in $W$ iff there is a path in $G$ from $v$ to some vertex in $F$.

For $v \in V \backslash F$, let $z_{v}=\sum_{\gamma \in \Gamma_{v}} y_{(v, \gamma)}^{\prime \prime}$. Note that by the constraints of the LP, for any vertex $v \in S$

$$
\begin{aligned}
\alpha(v) & =z_{v}-\sum_{v^{\prime} \in V \backslash F} \sum_{\gamma \in \Gamma_{v^{\prime}}} p_{\left(v^{\prime}, \gamma, v\right)} y_{\left(v^{\prime}, \gamma\right)}^{\prime \prime} \\
& \left.=z_{v}-\sum_{v^{\prime} \in S} \sum_{\gamma \in \Gamma_{v^{\prime}}} p_{\left(v^{\prime}, \gamma, v\right)} y_{\left(v^{\prime}, \gamma\right)}^{\prime \prime} \quad \text { (because all flow into } v \text { comes from } S\right) \\
& =z_{v}-\sum_{v^{\prime} \in S} \sum_{\gamma \in \Gamma_{v^{\prime}}} p_{\left(v^{\prime}, \gamma, v\right)} y_{\left(v^{\prime}, \gamma\right)}^{\prime \prime} \frac{z_{v^{\prime}}}{\sum_{\gamma^{\prime} \in \Gamma_{v^{\prime}}} y_{\left(v^{\prime}, \gamma^{\prime}\right)}^{\prime \prime}} \\
& =z_{v}-\sum_{v^{\prime} \in S} \sum_{\gamma \in \Gamma_{v^{\prime}}} p_{\left(v^{\prime}, \gamma, v\right)} \sigma\left(v^{\prime}\right)(\gamma) z_{v^{\prime}} \\
& =z_{v}-\sum_{v^{\prime} \in S} P_{v^{\prime}, v}^{\sigma} z_{v^{\prime}}
\end{aligned}
$$

Now, let us focus on the vertices in $W$. Note that, by definition, $W \subseteq S$. Consider the submatrix $P_{W, W}^{\sigma}$ obtained from $P^{\sigma}$ by eliminating the rows and columns whose indices are not in $W$. Note that since there is no flow into a vertex in $W$ from a vertex outside of $W$, the above equalities yield, for each $v \in W, \alpha(v)=z_{v}-\sum_{v \in W} P_{v^{\prime}, v}^{\sigma} z_{v^{\prime}}$. This can be written in matrix notation as $\left.\alpha^{T}\right|_{W}=\left.z^{T}\right|_{W}\left(I-P_{W, W}^{\sigma}\right)$.

Now, note that since every vertex in $W$ has a "flow" to $F$, in terms of the underlying Markov chain of the substochastic matrix $P_{W, W}^{\sigma}$, this means that every vertex in $W$ is transient, and that there is a power $d \geq 1$, such that $\left(P_{W, W}^{\sigma}\right)^{d}$ has the property that all its row sums are strictly less than 1 . Consequently, $\lim _{d \rightarrow \infty}\left(P_{W, W}^{\sigma}\right)^{d}=\mathbf{0}$ and the matrix $\left(I-P_{W, W}^{\sigma}\right)$ is invertible, with $\left.\left(I-P_{W, W}^{\sigma}\right)^{-1}=\sum_{i=0}^{\infty}\left(P_{W, W}^{\sigma}\right)^{i}\right)$, a nonnegative matrix. Thus, $\left.z^{T}\right|_{W}=\alpha^{T}\left(I-P_{W, W}^{\sigma}\right)^{-1}=\alpha^{T}\left(\sum_{i=0}^{\infty}\left(P_{W, W}^{\sigma}\right)^{i}\right.$. From this it follows, again because no vertex outside of $W$ has a flow into $W$, that for each $v \in W$ :

$$
\begin{aligned}
z_{v} & =\sum_{v^{\prime} \in V \backslash F} \alpha\left(v^{\prime}\right) \sum_{n=0}^{\infty} \sum_{\gamma \in \Gamma_{v}}\left(P^{\sigma}\right)_{v^{\prime}, v}^{n} \sigma(v)(\gamma) \\
& =\sum_{\gamma \in \Gamma_{v}} \sum_{v^{\prime} \in W} \alpha\left(v^{\prime}\right) \sum_{n=0}^{\infty}\left(P^{\sigma}\right)_{v^{\prime}, v}^{n} \sigma(v)(\gamma) \\
& \quad \sum_{\gamma \in \Gamma_{v}} y_{(v, \gamma)}^{\prime}
\end{aligned}
$$

where, in the last expression, the values $y_{(v, \gamma)}^{\prime}$, not to be mistaken with $y_{(v, \gamma)}^{\prime \prime}$, are values from the vector $y^{\prime}$ which we obtained in the proof that (1.) $\Rightarrow(2$.), from a given memoryless strategy $\sigma$. In this case, the strategy $\sigma$ in question is precisely the memoryless strategy we just defined based on $y^{\prime \prime}$. Thus, for all $v \in W$ :

$$
z_{v}=\sum_{\gamma \in \Gamma_{v}} y_{(v, \gamma)}^{\prime \prime}=\sum_{\gamma \in \Gamma_{v}} y_{(v, \gamma)}^{\prime}
$$


We next show that in fact for all $v \in W$ and $\gamma \in \Gamma_{v}, y_{(v, \gamma)}^{\prime \prime}=y_{(v, \gamma)}^{\prime}$. For $v \in W$ and $\gamma \in \Gamma_{v}$, we have:

$$
\begin{aligned}
y_{(v, \gamma)}^{\prime} & =\sum_{v^{\prime} \in W} \alpha\left(v^{\prime}\right) \sum_{i=0}^{\infty}\left(P_{W, W}^{\sigma}\right)_{v^{\prime}, v}^{i} \sigma(v)(\gamma) \\
& =\sum_{v^{\prime} \in W} \alpha\left(v^{\prime}\right) \sum_{i=0}^{\infty}\left(P_{W, W}^{\sigma}\right)_{v^{\prime}, v}^{i} \frac{y_{(v, \gamma)}^{\prime \prime}}{\sum_{\gamma^{\prime} \in \Gamma_{v}} y_{\left(v, \gamma^{\prime}\right)}^{\prime \prime}} \\
& =\frac{y_{(v, \gamma)}^{\prime \prime}}{\sum_{\gamma^{\prime} \in \Gamma_{v}} y_{\left(v, \gamma^{\prime}\right)}^{\prime \prime}} \sum_{v^{\prime} \in W} \alpha\left(v^{\prime}\right) \sum_{i=0}^{\infty}\left(P_{W, W}^{\sigma}\right)_{v^{\prime}, v}^{i}
\end{aligned}
$$

But recall that the "expected number of times we will transition out of state $v$ " is given by $\sum_{\gamma \in \Gamma_{v}} y_{(v, \gamma)}^{\prime}=\sum_{v^{\prime} \in W} \alpha\left(v^{\prime}\right) \sum_{i=0}^{\infty}\left(P_{W, W}^{\sigma}\right)_{v^{\prime}, v}^{i}$.

Hence $y_{(v, \gamma)}^{\prime}=\frac{y_{(v, \gamma)}^{\prime \prime}}{\sum_{\gamma^{\prime} \in \Gamma_{v}} y_{\left(v, \gamma^{\prime}\right)}^{\prime \prime}} \sum_{\gamma \in \Gamma_{v}} y_{(v, \gamma)}^{\prime}$. Thus, by using equation (3.1) and canceling, we get $y_{(v, \gamma)}^{\prime}=y_{(v, \gamma)}^{\prime \prime}$. Thus, since $y^{\prime \prime}$ is a feasible solution to the LP, we have that for any $v \in F:$

$$
\begin{aligned}
y_{v}^{\prime \prime} & =\sum_{v^{\prime} \in V \backslash F \gamma^{\prime} \in \Gamma_{v^{\prime}}} p_{\left(v^{\prime}, \gamma^{\prime}, v\right)} y_{\left(v^{\prime}, \gamma^{\prime}\right)}^{\prime \prime} \\
& \left.=\sum_{v^{\prime} \in W} \sum_{\gamma^{\prime} \in \Gamma_{v^{\prime}}} p_{\left(v^{\prime}, \gamma^{\prime}, v\right)} y_{\left(v^{\prime}, \gamma^{\prime}\right)}^{\prime \prime} \quad \text { (because all flow into } F \text { is from } W\right) \\
& =\sum_{v^{\prime} \in W} \sum_{\gamma^{\prime} \in \Gamma_{v^{\prime}}} p_{\left(v^{\prime}, \gamma^{\prime}, v\right)} y_{\left(v^{\prime}, \gamma^{\prime}\right)}^{\prime} \\
& =\operatorname{Pr}_{\alpha}^{\sigma}(\diamond\{v\})
\end{aligned}
$$

The last equality holds because, as we showed in the proof of $((1.) \Rightarrow(2)$.$) , the expression$ $\sum_{v^{\prime} \in W} \sum_{\gamma^{\prime} \in \Gamma_{v^{\prime}}} p_{\left(v^{\prime}, \gamma^{\prime}, v\right)} y_{\left(v^{\prime}, \gamma^{\prime}\right)}^{\prime}=\sum_{v^{\prime} \in V \backslash F} \sum_{\gamma^{\prime} \in \Gamma_{v^{\prime}}} p_{\left(v^{\prime}, \gamma^{\prime}, v\right)} y_{\left(v^{\prime}, \gamma^{\prime}\right)}^{\prime}$ is exactly the "expected number of times that we will visit the vertex $v \in F$ for the first time", which is precisely the probability $\operatorname{Pr}_{\alpha}^{\sigma}(\diamond\{v\})$.

Thus, clearly, $\sum_{v \in F_{i}} y_{v}^{\prime \prime}=\sum_{v \in F_{i}} \operatorname{Pr}_{\alpha}^{\sigma}(\diamond\{v\})=\operatorname{Pr}_{\alpha}^{\sigma}\left(\diamond F_{i}\right)$. Thus, since we have assumed that $\sum_{v \in F_{i}} y_{v}^{\prime \prime} \geq r_{i}$, we have established that $\operatorname{Pr}_{\alpha}^{\sigma}\left(\diamond F_{i}\right) \geq r_{i}$, for all target sets $F_{i}$.

This completes the proof that (2.) $\Rightarrow(1$.).

(3.) $\Leftrightarrow(1$.$) . Clearly (1.) \Rightarrow$ (3.), so we need to show that (3.) $\Rightarrow(1$.$) .$

Let $U$ be the set of achievable vectors, i.e., all $k$-vectors $r=\left\langle r_{1} \ldots r_{k}\right\rangle$ such that there is a (unrestricted) strategy $\sigma$ such that $\bigwedge_{i=1}^{k} \operatorname{Pr}_{\alpha}^{\sigma}\left(\diamond F_{i}\right) \geq r_{i}$. Let $U^{\odot}$ be the analogous set where the strategy $\sigma$ is restricted to be a possibly randomized but memoryless (stationary) strategy. Clearly, $U$ and $U^{\odot}$ are both downward closed, i.e., if $r \geq r^{\prime}$ and $r \in U$ then also $r^{\prime} \in U$, and similarly with $U^{\odot}$. Also, obviously $U^{\odot} \subseteq U$. We characterized $U^{\odot}$ in (1.) $\Leftrightarrow$ (2.), in terms of a multi-objective LP. Thus, $U^{\odot}$ is the projection of the feasible space of a set of linear inequalities (a polyhedral set), namely the set of inequalities in the variables $y$ given in Fig. 3 and the inequalities $\sum_{v \in F_{i}} y_{v} \geq r_{i}, i=1, \ldots, k$. The feasible space is a polyhedron in the space indexed by the $y$ variables and the $r_{i}$ 's, and $U^{\odot}$ is its projection on the subspace indexed by the $r_{i}$ 's. Since the projection of a convex set is convex, it follows that $U^{\odot}$ is convex. 
Suppose that there is a point $r \in U \backslash U^{\odot}$. Since $U^{\odot}$ is convex, this implies that there is a separating hyperplane (see, e.g., GLS93]) that separates $r$ from $U^{\odot}$, and in fact since $U^{\odot}$ is downward closed, there is a separating hyperplane with non-negative coefficients, i.e. there is a non-negative "weight" vector $w=\left\langle w_{1}, \ldots, w_{k}\right\rangle$ such that $w^{T} r=\sum_{i=1}^{k} w_{i} r_{i}>w^{T} x$ for every point $x \in U^{\odot}$.

Consider now the MDP $M$ with the following undiscounted reward structure. There is 0 reward for every state, action and transition, except for transitions to a state $v \in F$ from a state in $V \backslash F$; i.e. a reward is produced only once, in the first transition into a state of $F$. The reward for every transition to a state $v \in F$ is $\sum\left\{w_{i} \mid i \in\{1, \ldots, k\} \& v \in F_{i}\right\}$. By the definition, the expected reward of a policy $\sigma$ is $\sum_{i=1}^{k} w_{i} \operatorname{Pr}_{\alpha}^{\sigma}\left(\diamond F_{i}\right)$. From classical MDP theory, we know that there is a memoryless strategy (in fact even a deterministic one) that maximizes the expected reward for this type of reward structure. (Namely, this is a positive bounded reward case: see, e.g., Theorem 7.2.11 in Put94.) Therefore, $\max \left\{w^{T} x \mid x \in\right.$ $U\}=\max \left\{w^{T} x \mid x \in U^{\odot}\right\}$, contradicting our assumption that $w^{T} r>\max \left\{w^{T} x \mid x \in U^{\odot}\right\}$.

Corollary 3.5. Given an $M D P M=(V, \Gamma, \delta)$, a number of target sets $F_{i} \subseteq V, i=$ $1, \ldots, k+k^{\prime}$, such that every state $v \in F=\bigcup_{i=1}^{k+k^{\prime}} F_{i}$ is absorbing, and an initial state $u$ (or even initial distribution $\alpha \in \mathcal{D}(V))$ :

(a.) Given an extended achievability query for reachability, $\exists \sigma B$, where

$$
B \equiv \bigwedge_{i=1}^{k}\left(\operatorname{Pr}_{u}^{\sigma}\left(\diamond F_{i}\right) \geq r_{i}\right) \wedge \bigwedge_{j=k+1}^{k+k^{\prime}}\left(\operatorname{Pr}_{u}^{\sigma}\left(\diamond F_{j}\right)>r_{j}\right)
$$

we can in time polynomial in the size of the input, $|M|+|B|$, decide whether $\exists \sigma B$ is satisfiable and if so construct a memoryless strategy that satisfies it.

(b.) For $\epsilon>0$, we can compute an $\epsilon$-approximate Pareto curve $\mathcal{P}(\epsilon)$ for the multi-objective reachability problem with objectives $\diamond F_{i}, i=1, \ldots, k$, in time polynomial in $|M|$ and $1 / \epsilon$.

Proof. For (a.), consider the constraints of the LP in Figure 3, and add the following constraints: for each $i \in\{1, \ldots, k\}$ add the constraint $\sum_{v \in F_{i}} y_{v} \geq r_{i}$, and for each $j \in$ $\left\{k+1, \ldots, k+k^{\prime}\right\}$, add the constraint $\sum_{v \in F_{j}} y_{v} \geq r_{j}+z$, where $z$ is a new variable, and also add the constraint $z \geq 0$. Finally, consider the new objective "Maximize $z$ ". Solve this LP to find whether an optimal feasible solution $y^{*}, z^{*}$ exists, and if so whether $z^{*}>0$. If no solution exists, or if $z^{*} \leq 0$, then the extended achievability query is not satisfiable. Otherwise, if $z^{*}>0$, then a strategy that satisfies $\exists \sigma B$ exists, and moreover we can construct a memoryless strategy that satisfies it by using the vector $y^{\prime \prime}=y^{*}$ and picking the strategy $\sigma$ constructed from $y^{\prime \prime}$ in the proof of $(2.) \Rightarrow(1$.) in Theorem 3.2 .

Part (b.) is immediate from Theorem [3.2, and the results of [PY00], which show we can $\epsilon$-approximate the Pareto curve for multi-objective linear programs in time polynomial in the size of the constraints and objectives and in $1 / \epsilon$.

\section{Qualitative multi-OBJeCtive model CheCKInG}

Theorem 4.1. Given an $M D P M$, an initial state $u$, and a qualitative multi-objective query $B$, we can decide whether there exists a strategy $\sigma$ that satisfies $B$, and if so construct such 
a strategy, in time polynomial in $|M|$, and using only graph-theoretic methods (in particular, without linear programming).

Proof. By the discussion in Section 2, it suffices to consider the case where we are given an MDP, $M$, and two sets of $\omega$-regular properties $\Phi, \Psi$, and we want a strategy $\sigma$ such that

$$
\bigwedge_{\varphi \in \Phi} \operatorname{Pr}_{u}^{\sigma}(\varphi)=1 \wedge \bigwedge_{\psi \in \Psi} \operatorname{Pr}_{u}^{\sigma}(\psi)>0
$$

Assume the properties in $\Phi, \Psi$ are all given by (nondeterministic) Büchi automata $A_{i}$. We will use and build on results in [CY98. In CY98] (Lemma 4.4, page 1411) it is shown that we can construct from $M$ and from a collection $A_{i}, i=1, \ldots, m$, of Büchi automata, a new MDP $M^{\prime}$ (a refinement of $M$ ) which is the "product" of $M$ with the naive determinization of all the $A_{i}$ 's (i.e., the result of applying the standard subset construction on each $A_{i}$, without imposing any acceptance condition). Technically, we have to slightly adapt the constructions of [CY98, which use the convention that MDP states are either purely controlled or purely probabilistic, to the convention used in this paper which combines both control and probabilistic behavior at each state. But these adaptations are straightforward. For completeness, we recall the (adapted) formal definition of $M^{\prime}$. The states of the MDP $M^{\prime}$ are tuples $\left(x, z_{1}, \ldots, z_{m}\right)$, where $x$ is a state of the MDP, $M$, and $z_{i}$ is a set of states of $A_{i}$. The transition relation $\delta^{\prime}$ of $M^{\prime}$ is as follows. There exists a transition $\left(\left(x, z_{1}, \ldots, z_{m}\right), a, p,\left(x^{\prime}, z_{1}^{\prime}, \ldots, z_{m}^{\prime}\right)\right) \in \delta^{\prime}$ if and only if the transition $\left(x, a, p, x^{\prime}\right)$ is in $M$ and, for each $i=1, \ldots, m, z_{i}^{\prime}$ is precisely the set of states in the Büchi automaton $A_{i}$ that one could reach with one transition, starting from some state in the set $z_{i}$ and reading the symbol $l\left(x^{\prime}\right)$. Technically, we also have to add a dummy initial state $x_{0}$ to the $\operatorname{MDP}, M$, such that there is a single enabled action, $\gamma_{0}$, at $x_{0}$, and such that there are transitions from $x_{0}$ on action $\gamma_{0}$ to other states according to some initial probability distribution on states, $\alpha \in \mathcal{D}(V)$. Thus, in particular, if we assume there is just one initial state $u$ in the MDP, $M$, then we would now have one transition $\left(x_{0}, \gamma_{0}, 1, u\right) \in \delta$ in the new $M$ with added dummy state $x_{0}$. The reason for adding the dummy $x_{0}$ is because our definition of the product $M^{\prime}$ does not use the label of the initial state in defining the transitions of $M^{\prime}$. We also assume, w.l.o.g., that each Büchi automaton $A_{i}$ has a single initial state $s_{0}^{i}$. In this way, the initial state of $M^{\prime}$ becomes the tuple $v_{0}=\left(x_{0},\left\{s_{0}^{1}\right\}, \ldots,\left\{s_{0}^{m}\right\}\right)$.

By Lemma 4.4 and 4.5 of [CY98], this MDP $M^{\prime}$ has the following two properties. For every subset $R$ of $\Phi \cup \Psi$ there is a subset $T_{R}$ of corresponding "target states" of $M^{\prime}$ (and we can compute this subset efficiently, in time polynomial in the size of $M^{\prime}$ ) that satisfies the following two conditions:

(I) If a trajectory of $M^{\prime}$ hits a state in $T_{R}$ at some point, then we can apply from that point on a strategy $\mu_{R}$ (which is deterministic but uses memory) which ensures that the resulting infinite trajectory satisfies all properties in $R$ almost surely (i.e., with conditional probability 1 , conditioned on the initial prefix that hits $T_{R}$ ).

(II) For every strategy, the set of trajectories that satisfy all properties in $R$ and do not infinitely often hit some state of $T_{R}$ has probability 0 .

We now outline the algorithm for deciding qualitative multi-objective queries.

(1) Construct the MDP $M^{\prime}$ from $M$ and from the properties $\Phi$ and $\Psi$ (in other words, using one automaton for each property in $\Phi$ and one for each property in $\Psi$ ). 
(2) Compute $T_{\Phi}$, and compute for each property $\psi_{i} \in \Psi$ the set of states $T_{R_{i}}$ where $R_{i}=$ $\Phi \cup\left\{\psi_{i}\right\}$

(3) If $\Phi \neq \emptyset$, prune $M^{\prime}$ by identifying and removing all "bad" states by applying the following rules.

(a) All states $v$ that cannot "reach" any state in $T_{\Phi}$ are "bad" 4

(b) If for a state $v$ there is an action $\gamma \in \Gamma_{v}$ such that there is a transition $\left(v, \gamma, p, v^{\prime}\right) \in$ $\delta^{\prime}, p>0$, and $v^{\prime}$ is bad, then remove $\gamma$ from $\Gamma_{v}$.

(c) If for some state $v, \Gamma_{v}=\emptyset$, then mark $v$ as bad.

Keep applying these rules until no more states can be labelled bad and no more actions removed for any state.

(4) Restrict $M^{\prime}$ to the reachable states (from the initial state $v_{0}$ ) that are not bad, and restrict their action sets to actions that have not been removed, and let $M^{\prime \prime}$ be the resulting MDP.

(5) If $\left(M^{\prime \prime}=\emptyset\right.$ or $\exists \psi_{i} \in \Psi$ such that $M^{\prime \prime}$ does not contain any state of $\left.T_{R_{i}}\right)$ then return No.

Else return Yes.

Correctness proof: In one direction, suppose there is a strategy $\sigma$ such that $\bigwedge_{\varphi \in \Phi} \operatorname{Pr}_{u}^{\sigma}(\varphi)=$ $1 \wedge \bigwedge_{\psi \in \Psi} \operatorname{Pr}_{u}^{\sigma}(\psi)>0$. First, note that there cannot be any finite prefix of a trajectory under $\sigma$ that hits a state that cannot reach any state in $T_{\Phi}$. For, if there was such a path, then all trajectories that start with this prefix would go only finitely often through $T_{\Phi}$. Hence (by property (II) above) almost all these trajectories do not satisfy all properties in $\Phi$, which contradicts the fact that all these properties have probability 1 under $\sigma$. From the fact that no path under $\sigma$ hits a state that cannot reach $T_{\Phi}$, it follows by an easy induction that no finite trajectory under $\sigma$ hits any bad state. That is, under $\sigma$ all trajectories stay in the sub-MDP $M^{\prime \prime}$. Since every property $\psi_{i} \in \Psi$ has probability $\operatorname{Pr}_{u}^{\sigma}\left(\psi_{i}\right)>0$ and almost all trajectories that satisfy $\psi_{i}$ and $\Phi$ must hit a state of $T_{R_{i}}$ (property (II) above), it follows that $M^{\prime \prime}$ contains some state of $T_{R_{i}}$ for each $\psi_{i} \in \Psi$. Thus the algorithm returns Yes.

In the other direction, suppose that the algorithm returns Yes. First, note that for all states $v$ of $M^{\prime \prime}$, and all enabled actions $\gamma \in \Gamma_{v}$ in $M^{\prime \prime}$, all transitions $\left(v, \gamma, p, v^{\prime}\right) \in \delta, p>0$ of $M^{\prime}$ must still be in $M^{\prime \prime}$ (otherwise, $\gamma$ would have been removed from $\Gamma_{v}$ at some stage using rule $3(\mathrm{~b})$ ). On the other hand, some states may have some missing actions in $M^{\prime \prime}$. Next, note that all bottom strongly connected components (BSCCs) of $M^{\prime \prime}$ (to be more precise, in the underlying one-step reachability graph of $M^{\prime \prime}$ ) contain a state of $T_{\Phi}$ (if $\Phi=\emptyset$ then all states are in $T_{\Phi}$ ), for otherwise the states in these BSCCs would have been eliminated at some stage using rule $3(\mathrm{a})$.

Define the following strategy $\sigma$ which works in two phases. In the first phase, the trajectory stays within $M^{\prime \prime}$. At each control state take a random action that remains in $M^{\prime \prime}$ out of the state; the probabilities do not matter, we can use any non-zero probability for all the remaining actions. In addition, at each state, if the state is in $T_{\Phi}$ or it is in $T_{R_{i}}$ for some property $\psi_{i} \in \Psi$, then with some nonzero probability the strategy decides to terminate phase 1 and move to phase 2 by switching to the strategy $\mu_{\Phi}$ or $\mu_{R_{i}}$ respectively,

\footnotetext{
${ }^{3}$ Actually these sets can all be computed together: we can compute maximal closed components of the MDP, determine the properties that each component favors (see Def. 4.1 of [CY98]), and tag each state with the sets for which it is a target state.

${ }^{4} \mathrm{By}$ "reach", we mean that starting at the state $v=v_{0}$, there a sequence of transitions $\left(v_{i}, \gamma, p_{i}, v_{i+1}\right) \in \delta$, $p_{i}>0$, such that $v_{n} \in T_{\Phi}$ for some $n \geq 0$.
} 
which it applies from that point on. (Note: a state may belong to several $T_{R_{i}}$ 's, in which case each one of them gets some non-zero probability - the precise value is unimportant.)

We claim that this strategy $\sigma$ meets the desired requirements - it ensures probability 1 for all properties in $\Phi$ and positive probability for all properties in $\Psi$. For each $\psi_{i} \in \Psi$, the MDP $M^{\prime \prime}$ contains some state of $T_{R_{i}}$; with nonzero probability the process will follow a path to that state and then switch to the strategy $\mu_{R_{i}}$ from that point on, in which case it will satisfy $\psi_{i}$ (property (I) above). Thus, all properties in $\Psi$ are satisfied with positive probability.

As for $\Phi$ (if $\Phi \neq \emptyset$ ), note that with probability 1 the process will switch at some point to phase 2 , because all BSCCs of $M^{\prime \prime}$ have a state in $T_{\Phi}$. When it switches to phase 2 it applies strategy $\mu_{\Phi}$ or $\mu_{R_{i}}$ for some $R_{i}=\Phi \cup\left\{\psi_{i}\right\}$, hence in either case it will satisfy all properties of $\Phi$ with probability 1 .

\section{Quantitative multi-objective model CheCKing.}

\section{Theorem 5.1.}

(1.) Given an $M D P M$, an initial state $u$, and a quantitative multi-objective query $B$, we can decide whether there exists a strategy $\sigma$ that satisfies $B$, and if so construct such a strategy, in time polynomial in $|M|$.

(2.) Moreover, given $\omega$-regular properties $\Phi=\left\langle\varphi_{1}, \ldots, \varphi_{k}\right\rangle$, we can construct an $\epsilon$-approximate Pareto curve $P_{M_{u}, \Phi}(\epsilon)$, for the set of achievable probability vectors $U_{M_{u}, \Phi}$ in time polynomial in $M$ and in $1 / \epsilon$.

Proof. For (1.), by the discussion in Section 2, we only need to consider extended achievability queries, $B \equiv \bigwedge_{i=1}^{k^{\prime}} \operatorname{Pr}_{u}^{\sigma}\left(\varphi_{i}\right) \geq r_{i} \wedge \bigwedge_{j=k^{\prime}+1}^{k} \operatorname{Pr}_{u}^{\sigma}\left(\varphi_{j}\right)>r_{j}$, where $k \geq k^{\prime} \geq 0$, and for a vector $r \in(0,1]^{k}$. Let $\Phi=\left\langle\varphi_{1}, \ldots, \varphi_{k}\right\rangle$. We are going to reduce this multi-objective problem with objectives $\Phi$ to the quantitative multi-objective reachability problem studied in Section 3, From our reduction, both (1.) and (2.) will follow, using Corollary 3.5, As in the proof of Theorem 4.1, we will build on constructions from CY98: form the MDP $M^{\prime}$ consisting of the product of $M$ with the naive determinizations of the automata $A_{i}$ for the properties $\varphi_{i} \in \Phi$. For each subset $R \subseteq \Phi$ we determine the corresponding subset $T_{R}$ of target states in $M^{\prime}$ 占

Construct the following MDP $M^{\prime \prime}$. Add to $M^{\prime}$ a new absorbing state $s_{R}$ for each subset $R$ of $\Phi$. For each state $u$ of $M^{\prime}$ and each maximal subset $R$ such that $u \in T_{R}$ add a new action $\gamma_{R}$ to $\Gamma_{u}$, and a new transition $\left(u, \gamma_{R}, 1, s_{R}\right)$ to $\delta$. With each property $\varphi_{i} \in \Phi$ we associate the subset of states $F_{i}=\left\{s_{R} \mid \varphi_{i} \in R\right\}$. Let $\bar{F}=\left\langle\diamond F_{1}, \ldots, \diamond F_{k}\right\rangle$. Let $u^{*}$ be the initial state of the product MDP $M^{\prime \prime}$, given by the start state $u$ of $M$ and the start states of all the naively determinized $A_{i}$ 's. Recall that $U_{M_{u}, \Phi} \subseteq[0,1]^{k}$ denotes the achievable set for the properties $\Phi$ in $M$ starting at $u$, and that $U_{M_{u^{*}}^{\prime \prime}} \bar{F}$ denotes the achievable set for $\bar{F}$ in $M^{\prime \prime}$ starting at $u^{*}$.

Lemma 5.2. $U_{M_{u}, \Phi}=U_{M_{u^{*}}^{\prime \prime}, \bar{F}}$. Moreover, from a strategy $\sigma$ that achieves $r$ in $U_{M_{u}, \Phi}$, we can recover a strategy $\sigma^{\prime}$ that achieves $r$ in $U_{M_{u^{*}}^{\prime \prime}, \bar{F}}$, and vice versa.

\footnotetext{
${ }^{5}$ Again, we don't need to compute these sets separately. See Footnote 3.
} 
Proof. One direction is easy. Given such a strategy $\sigma^{\prime}$ in $M^{\prime \prime}$, we follow in $M^{\prime}$ (and in $M$ ) the same strategy (of course, only the first component of states of $M^{\prime \prime}$ matters in $M)$, until just before it transitions to a state $s_{R}$, at which point it must be in $T_{R}$, and at that point our strategy $\sigma$ switches to the strategy $\mu_{R}$. This guarantees, for every $\varphi_{i} \in \Phi$, $\operatorname{Pr}_{u}^{\sigma}\left(\varphi_{i}\right) \geq \operatorname{Pr}_{u^{*}}^{\sigma^{\prime}}\left(\diamond F_{i}\right) \geq r_{i}$.

For the other direction, suppose that the claim is not true, i.e. there is a strategy $\sigma$ in $M$ which ensures probability $\operatorname{Pr}_{u}^{\sigma}\left(\varphi_{i}\right) \geq r_{i}, i=1, \ldots, k$, but $r \notin U_{M_{u^{*}}^{\prime \prime}, \bar{F}}$. Note that all states in $F=\cup_{i=1}^{k} F_{i}$ are absorbing. From Theorem 3.2 we know that $U_{M_{u^{*}}^{\prime \prime}, \bar{F}}=U_{M_{u^{*}}^{\prime \prime}, \bar{F}}^{\odot}$ where $U_{M_{u^{*}}^{\prime \prime}, \bar{F}}^{\odot}$ is the set of value vectors achievable by memoryless strategies. Recall, that $U_{M_{u^{*}}^{\prime \prime}, \bar{F}}=U_{M_{u^{*}}^{\prime \prime}, \bar{F}}^{\odot}$ is convex, and that it is downward-closed. Since $r \notin U_{M_{u^{*}}^{\prime \prime}, \bar{F}}$, as in the proof of $(3.) \Rightarrow(1$.$) in Thm. 3.2, there must be a separating hyperplane, i.e., a non-$ negative weight vector $w=\left\langle w_{1}, \ldots, w_{k}\right\rangle$ such that $w^{T} r=\sum_{i=1}^{k} w_{i} r_{i}>w^{T} x$ for every point $x \in U_{M_{u^{*}}^{\prime \prime}, \bar{F}}$.

Consider $M$ with the following reward structure, denoted rew $(w)$ : a trajectory $\tau$ of $M$ receives reward $\sum\left\{w_{i} \mid \tau\right.$ satisfies $\left.\varphi_{i}\right\}$. This is not the traditional type of reward structure where reward is obtained at the states and transitions of the trajectory; it is obtained only at infinity when the trajectory has finished and we get a reward that depends on the properties that were satisfied. In [CY98, optimization of the expected reward for MDPs with this kind of reward structure was studied and solved by reducing the problem to an MDP with a classical type of reward. We reuse that construction here. Consider the MDP $M^{\prime \prime}$ augmented with a traditional type of reward structure, denoted $r e w^{\prime \prime}$, in which each transition of the form $\left(u, \gamma_{R}, 1, s_{R}\right)$ produces reward $\sum\left\{w_{i} \mid \varphi_{i} \in R\right\}$, while all other transitions (and states and actions) give 0 reward. Let $\hat{M}^{\prime \prime}$ be a subMDP of $M^{\prime \prime}$ that contains for each state $u$ only one (at most) transition of the form $\left(u, \gamma_{R}, 1, s_{R}\right)$, namely the one that produces the maximum reward (breaking ties arbitrarily). Clearly, there is no reason ever to select from a state $u$ any transition $\left(u, \gamma_{R^{\prime}}, 1, s_{R^{\prime}}\right)$ that produces lower reward, thus, $M^{\prime \prime}$ and $\hat{M}^{\prime \prime}$ have the same optimal expected reward. It is shown in CY98 that the optimal expected rewards in $(M, r e w(w))$ and $\left(\hat{M}^{\prime \prime}, r e w^{\prime \prime}\right)$, and thus also in $\left(M^{\prime \prime}, r e w^{\prime \prime}\right)$, are equal to each other. Moreover, the optimum value in these MDPs is achievable, i.e., there are optimal strategies, and in fact a deterministic finite-memory optimal strategy can be constructed.

The optimal expected reward in $(M, \operatorname{rew}(w))$ is at least $w^{T} r$ (because strategy $\sigma$ achieves $\left.w^{T} r\right)$, while the optimal expected reward in $\left(M^{\prime \prime}, r e w^{\prime \prime}\right)$ is equal to $\max \left\{w^{T} x \mid\right.$ $\left.x \in U_{M_{u^{*}}^{\prime \prime}, \bar{F}}\right\}$, because rewards are only obtained by transitioning to a state in $F$. Therefore, $w^{T} r \leq \max \left\{w^{T} x \mid x \in U_{M_{u^{*}}^{\prime \prime}, \bar{F}}\right\}$, contradicting our hypothesis that $w^{T} r>\max \left\{w^{T} x \mid\right.$ $\left.x \in U_{M_{u^{*}}^{\prime \prime}, \bar{F}}\right\}$.

It follows from the lemma that: there exists a strategy $\sigma$ in $M$ such that

$$
\bigwedge_{i=1}^{k^{\prime}} \operatorname{Pr}_{u}^{\sigma}\left(\varphi_{i}\right) \geq r_{i} \wedge \bigwedge_{j=k^{\prime}+1}^{k} \operatorname{Pr}_{u}^{\sigma}\left(\varphi_{j}\right)>r_{j}
$$


if and only if there exists a strategy $\sigma^{\prime}$ in $M^{\prime \prime}$ such that

$$
\bigwedge_{i=1}^{k^{\prime}} \operatorname{Pr}_{u^{*}}^{\sigma}\left(\diamond F_{i}\right) \geq r_{i} \wedge \bigwedge_{j=k^{\prime}+1}^{k} \operatorname{Pr}_{u^{*}}^{\sigma}\left(\diamond F_{j}\right)>r_{j}
$$

Moreover, such strategies can be recovered from each other. Thus (1.) and (2.) follow, using Corollary 3.5 .

\section{Concluding Remarks}

We mention that recent results by Diakonikolas and Yannakakis [DY08] provide improved upper bounds for appoximation of convex Pareto curves, and for computing a smallest such approximate convex Pareto set. These results yield significantly improved algorithms, particularly in the bi-objective case, for the multi-objective LP problem, and thus also for the multi-objective MDP problems studied in this paper. In particular, in the bi-objective MDP case, DY08 provides a polynomial time algorithm to compute a minimal $\epsilon$-approximate (convex) Pareto set (i.e., one with the fewest number of points possible).

We mention that, although we use LP methods to obtain our complexity upper bounds, in practice there is a way to combine other efficient iterative methods used for solving MDPs, e.g., based on value iteration or policy (strategy) iteration, with our results in order to approximate the Pareto curve for multi-objective model checking. This is because the results of [PY00, DY08] for multi-objective convex optimization problems only require a blackbox routine that optimizes (exactly or approximately) positive linear combinations of the objectives. Specifically, in our setting the multiple MDP objectives ask to optimize the probabilities of different linear-time $\omega$-regular properties. By using the results in [CY98, it is possible to reduce the problem of optimizing such positive linear combinations to the problem of finding the optimal expected reward for a new MDP with positive rewards. The task of computing or approximating this optimal expected reward can be carried out using any of various standard iterative methods, e.g., based on value iteration and policy iteration (see [Put94]). These can thus be used to answer (exactly or approximately) the black-box queries required by the methods of [PY00, DY08], thereby yielding a method for approximating the Pareto curve (albeit, without the same theoretical complexity guarantees).

An important extension of the applications of our results is to extend the asymmetric assume-guarantee compositional reasoning rule discussed in Section 2 to a general compositional framework for probabilistic systems. It is indeed possible to describe symmetric assume-guarantee rules that allow for general composition of MDPs. A full treatment of the general compositional framework requires a separate paper, and we plan to expand on this in follow-up work.

Acknowledgements. We thank the Newton Institute, where we initiated discussions on the topics of this paper during the Spring 2006 programme on Logic and Algorithms. Several authors acknowledge support from the following grants: EPSRC GR/S11107 and EP/D07956X, MRL 2005-04; NSF grants CCR-9988322, CCR-0124077, CCR-0311326, and ANI-0216467, BSF grant 9800096, Texas ATP grant 003604-0058-2003, Guggenheim Fellowship; NSF CCF-04-30946 and NSF CCF-0728736. 


\section{REFERENCES}

[Car83] P. Carstensen. Complexity of some parametric integer and network programming problems. Mathematical Programming, 26(1):64-75, 1983.

[Clí97] J. Clímaco, editor. Multicriteria Analysis. Springer-Verlag, 1997.

[CMH06] K. Chatterjee, R. Majumdar, and T. Henzinger. Markov decision processes with multiple objectives. In Proc. of 23rd Symp. on Theoretical Aspects of Computer Science, volume LNCS 3884, pages $325-336,2006$.

[CY95] C. Courcoubetis and M. Yannakakis. The complexity of probabilistic verification. Journal of the ACM, 42(4):857-907, 1995.

[CY98] C. Courcoubetis and M. Yannakakis. Markov decision processes and regular events. IEEE Trans. on Automatic Control, 43(10):1399-1418, 1998.

[DY08] I. Diakonikolas and M. Yannakakis. Succinct Approximate Convex Pareto Curves. In Proc. of ACM-SIAM Symp. on Discrete Algorithms (SODA'08), 2008.

[Ehr05] M. Ehrgott. Multicriteria optimization. Springer-Verlag, 2005.

[Fur80] N. Furukawa. Characterization of optimal policies in vector-valued Markovian decision processes. Mathematics of Operations Research, 5(2):271-279, 1980.

[Gho90] M. K. Ghosh. Markov decision processes with multiple costs. Oper. Res. Lett., 9(4):257-260, 1990.

[GLS93] M. Grötschel, L. Lovász, and A. Schrijver. Geometric Algorithms and Combinatorial Optimization. Springer-Verlag, 2nd edition, 1993.

[Hen83] M. I. Henig. Vector-valued dynamic programming. SIAM J. Control Optim., 21(3):490-499, 1983.

[MS01] K. Mulmuley and P. Shah. A lower bound for the shortest path problem. J. Comput. System Sci., 63(2):253-267, 2001.

[Put94] M. L. Puterman. Markov Decision Processes. Wiley, 1994.

[PY00] C. Papadimitriou and M. Yannakakis. On the approximability of trade-offs and optimal access of web sources. In Proc. of 41st IEEE Symp. on Foundations of Computer Science, pages 86-92, 2000 .

[Var85] M. Vardi. Automatic verification of probabilistic concurrent finite-state programs. In Proc. of 26th IEEE FOCS, pages 327-338, 1985.

[Whi82] D. J. White. Multi-objective infinite-horizon discounted Markov decision processes. J. Math. Anal. Appl., 89(2):639-647, 1982.

[WT98] K. Wakuta and K. Togawa. Solution procedures for multi-objective Markov decision processes. Optimization. A Journal of Mathematical Programming and Operations Research, 43(1):29-46, 1998. 